\title{
Occult senile cardiac amyloid in severe calcific aortic stenosis is not rare and has a poor prognosis: a 146 patient CMR biopsy study
}

\author{
Thomas A Treibel ${ }^{1,2^{*}}$, Marianna Fontana ${ }^{3,2}$, Janet A Gilbertson ${ }^{3}$, Karen A Boniface ${ }^{3}$, Steven K White ${ }^{1,2}$, \\ Amna Abdel-Gadir ${ }^{1,2}$, Stefania Rosmini ${ }^{1,3}$, David F Hutt ${ }^{3}$, Carol J Whelan ${ }^{3}$, Julian D Gillmore ${ }^{3}$, \\ Ashutosh Wechalekar ${ }^{3}$, Martin P Hayward ${ }^{2}$, Michael A Ashworth ${ }^{4}$, Philip N Hawkins' ${ }^{3}$, James C Moon ${ }^{1,2}$
}

From 19th Annual SCMR Scientific Sessions

Los Angeles, CA, USA. 27-30 January 2016

\section{Background}

Severe degenerative calcific aortic stenosis (cAS) affects $3 \%$ of the over 75 year-olds and leads to heart failure and death unless the valve is replaced. Coexisting cardiac amyloid - typically wild-type Transthyretin (wtATTR) has been reported but has not been systematically studied: its prevalence and prognostic significance are unknown. Multiparametric CMR (function, LGE, T1 and ECV mapping) has high sensitivity and specificity for cardiac amyloidosis. We sought, in cAS, to define the prevalence of occult cardiac amyloid by biopsy, subtype it, explore its CMR presentation and understand its prognostic significance.

\section{Methods}

As part of the RELIEF-AS study (NCT 02174471), 146 patients with severe AS awaiting aortic valve replacement (AVR) underwent CMR (LGE, T1 mapping) and intra-operative myocardial biopsies. 108 patients had calcific AS (cAS) [75 \pm 6 years; $58 \%$ male]; the remainder had bicuspid (36), rheumatic (1) or unicuspid AS (1). Biopsies were screened for cardiac amyloid by congo red staining; if positive, tissue was fully subtyped (immunohistochemistry, mass spectrometry as needed), and patients underwent full clinical amyloid assessment including genotyping and DPD (bone tracer) scintigraphy.

\section{Results}

Myocardial biopsy identified amyloid depositions in 6 out of 108 cAS patients (all age $>65$ years); none in the other cohorts. All were TTR. All were by genotyping wild-type. Two had definite cardiac amyloidosis by CMR and DPD, but were not recognized by routine preoperative work-up (Figure 1). Four other patients had findings by multiparametric CMR that could be explained solely by severe AS (Table 1 ). At median follow-up of 1.9 years (0.4-4.2 years), $50 \%$ of the affected patients had died compared to $7.8 \%$ in the remaining cAS cohort. This was highly significant and, of all parameters assessed, the presence of TTR amyloid had the highest hazard ratio for death (HR 9.4 [2.4-35.6], $p=$ 0.001, univariable Cox regression analysis).

\section{Conclusions}

Occult cardiac amyloid (wild-type TTR) has a prevalence of $6 \%$ in cAS undergoing surgical AVR (mean age 75). It has a poor outcome even when at low levels of infiltration. Multiparametric CMR becomes diagnostic in $1 / 3$, but early disease looks like the changes of AS here, DPD scanning adds value.

\footnotetext{
Authors' details

${ }^{1}$ Cardiac Imaging, Barts Heart Centre, London, UK. ${ }^{2}$ Institute of Cardiovascular Sciences, University College London, London, UK. ${ }^{3}$ National Amyloidosis Centre, Royal Free Campus, University College London, London, UK. ${ }^{4}$ Department of Histopathology, Great Ormond Street Hospital for Children, London, UK.
} 


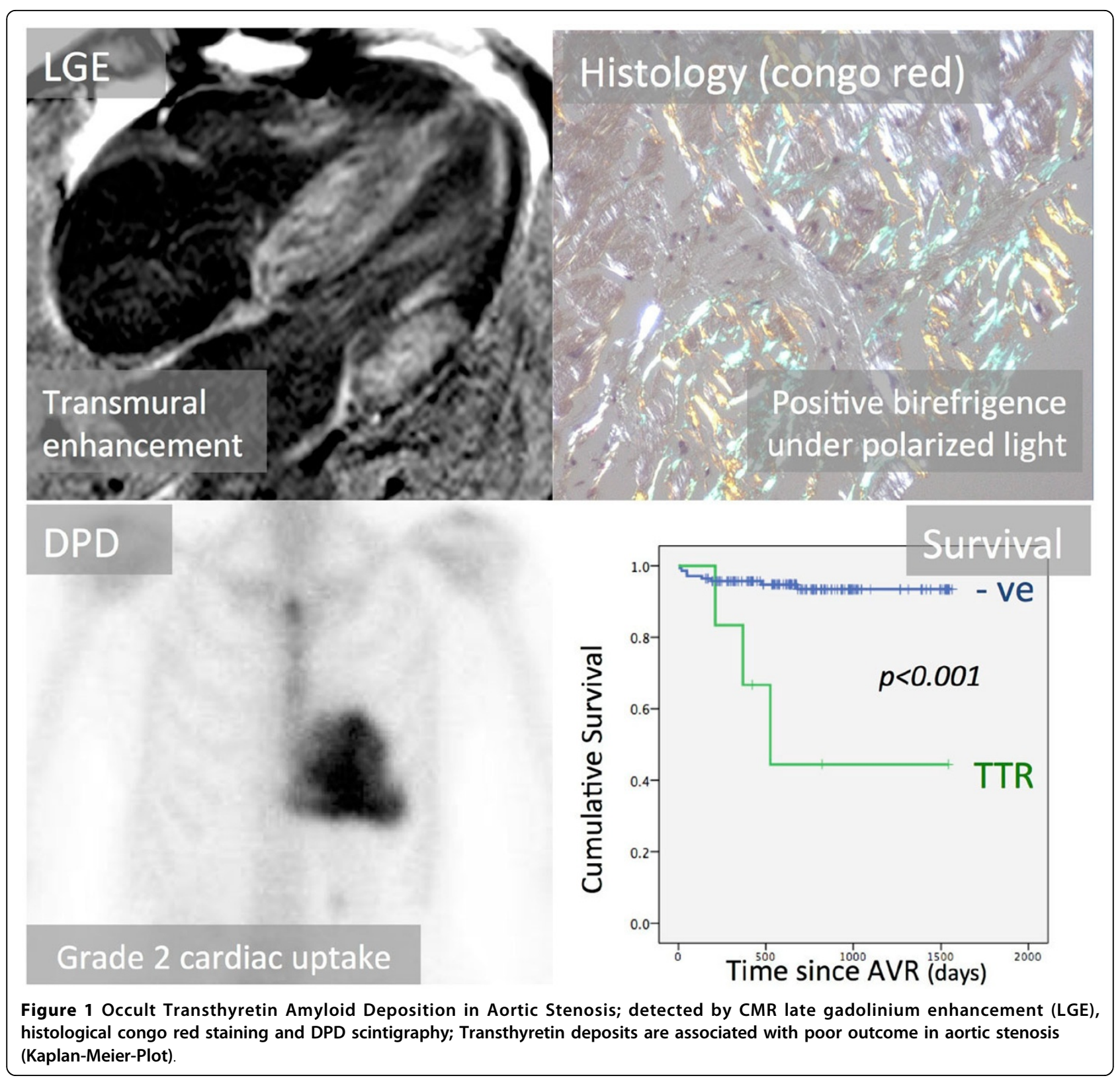

Table 1 Pre-operative characteristics of patients with TTR on cardiac biopsy

\begin{tabular}{|c|c|c|c|c|c|c|}
\hline & Patient 1 & Patient 2 & Patient 3 & Patient 4 & Patient 5 & Patient 6 \\
\hline Age/Gender & 73 female & 69 male & 80 female & 85 male & 84 male & 71 male \\
\hline Biopsy/Genotype & TTR/wild-type & TTR/wild-type & TTR/wild-type & TTR/wild-type & TTR/wild-type & TTR/wild-type \\
\hline CMR LGE pattern* & Amyloidosis & Amyloidosis & AS & AS & AS & AS \\
\hline ECV & $60 \%$ & $52 \%$ & $31 \%$ & $25 \%$ & $32 \%$ & $32 \%$ \\
\hline LV mass index $\left(\mathrm{g} / \mathrm{m}^{2}\right)$ & 137 & 150 & 117 & 101 & 93 & 132 \\
\hline DPD Scintigraphy & Grade 2 & Grade 2 & NA & Grade 1 & NA & Grade 1 \\
\hline Peak AV Gradient $(\mathrm{mmHg})$ & 74 & 45 & 70 & 110 & 61 & 116 \\
\hline AVAi $\left(\mathrm{cm}^{2} / \mathrm{m}^{2}\right)$ & 0.36 & 0.52 & 0.6 & 0.34 & 0.35 & 0.24 \\
\hline Follow-up Status & Alive & Dead & Dead & Alive & Dead & Alive \\
\hline
\end{tabular}

*LGE pattern consistent with diagnosis of cardiac amyloidosis / aortic stenosis (AS) 
doi:10.1186/1532-429X-18-S1-040

Cite this article as: Treibel et al: Occult senile cardiac amyloid in severe calcific aortic stenosis is not rare and has a poor prognosis: a 146 patient CMR biopsy study. Journal of Cardiovascular Magnetic Resonance 2016 18(Suppl 1):040.

Submit your next manuscript to BioMed Central and take full advantage of:

- Convenient online submission

- Thorough peer review

- No space constraints or color figure charges

- Immediate publication on acceptance

- Inclusion in PubMed, CAS, Scopus and Google Scholar

- Research which is freely available for redistribution 